\title{
Basic Science Symposium I: Bone Graft Substitutes
}

\author{
Basic Science Symposia Deputy Editor: Vijay K. Goel, PhD \\ Bone Graft Substitutes Symposium Editor: Celeste Abjornson, PhD
}

PANEL MEMBERS:

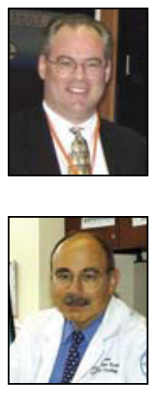

Todd Boyce, PhD

Osteotech, Inc.,

Eatontown, New Jersey

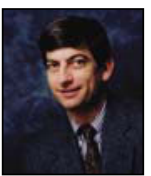

Scott D. Boden, MD

The Emory Orthopaedics \& Spine Center

Emory University School of Medicine

Atlanta, Georgia

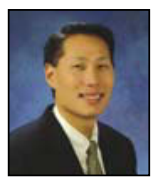

Jeffrey C. Wang, MD

UCLA Comprehensive Spine Center

Santa Monica, California

\section{INTRODUCTION}

by Celeste Abjornson, PhD, and Vijay K. Goel, PhD

Bone graft substitutes is a general term to describe any material used to aid in regeneration of bone, such as in a fracture, or in promotion of bone, such as in a spine fusion. There is a wide spectrum of materials used today for the purpose of grafting; however, their ultimate goal remains the same-to form functionally viable bone that meets the needs of the site.

The first known bone grafting procedure documented in modern medicine was described in the early nineteenth century and used what is still considered by some the gold standard, autograft. In the early 1990s, demineralized bone matrix and processed structural allografts became commercially available. In 2002, the first bone morphogenic protein became available with the FDA clearance of rhBMP-2 on a type-I collagen sponge in conjunction with a tapered, threaded intervertebral fusion cage (LT-Cage; Medtronic Sofamor Danek, Minneapolis, Minnesota) for the indication of degenerative lumbar disc disease.

Today, there is a plethora of options available-calcium-based, collagen-based, polymer, allograft, synthetic proteins-and the list goes on. But the difficulty lies in applying these materials in the right indications. Spine surgery is also in a period of great flux with new technologies exploding into the market. The question of how to pair these new materials for grafting with these surgical options is largely not well understood. The goal of this symposium is to ask experts in the field of bone graft substitutes what the standards of today are and how they should be applied to the technologies of tomorrow. We would like to thank our panel for their interesting and candid answers to the thought-provoking questions below. 
QUESTION \#1 What is an ideal bone graft? Is autograft still the standard of care? If using an osteoconductiveonly material, which adjuncts (ie, platelet-rich plasma, bone marrow aspirate, etc.) do you believe are the most effective?

Boyce: First a caveat: I'm not a clinician. I offer the perspective of a researcher who has academic training and corporate experience. That being said, I believe that the ideal bone graft depends upon the environment. Posterolateral fusion is different from interbody fusion, and both of these are different from the situation where there is a bony void surrounded by marrow and bone.

In general, I believe that surgeons should prefer to combine grafting materials in order to achieve a combination of matrix or scaffold, plus growth factors/BMPs, plus cells. The matrix is osteoconductive bone void filler or some similar material. Factors, used to signal to cells, are an osteoinductive material such as demineralized bone or a recombinant growth factor; and cells would come from bone marrow, local bone, or autograft.
There are many available options to achieve this combination, with some components being more effective than others.

I do believe that autograft is still the de facto standard, primarily because performance is still measured against it. However, it's also clear that it is possible to exceed the performance of autograft, at least in some circumstances. Osteoconductive materials clearly can benefit from the addition of cells that are either osteogenic or can differentiate into osteogenic cells. The difficulty lies in obtaining cells with the right lineage in great enough numbers to be effective. Many of us are familiar with the variability in cell type and number that can exist when collecting bone marrow. When it is feasible, the addition of an inductive material can supplement the cell addition.
Lane: The ideal bone graft is a material that contains a number of components. It should provide a playing field or an osteoconductive surface upon which bone is easily made along a predetermined orientation. Secondly, it should contain cells that can carry out a particular process; these are usually called stem cells. Thirdly, it should contain factors or biological components that drive the process.

Autogenous graft was considered the standard of care, but in reality it no longer is the leader. Studies have been performed, for instance, in interbody fusion using BMP alone against autogenous graft, and with adequate numbers BMP actually had superior results. Autogenous graft does contain many of the components, but it was found in the body - but not created for this specific purpose. I think that you could maximize the specific components of a potential graft, and it would be superior ultimately to autogenous graft.
Now if you're going to use osteoconductive-only material, that will not suffice, because you're going to need to add factors obtained either from the local environment or brought into the area, and you're going to also require cells that will do the job. Recent information has now shown that the stem cells that may contribute to the repair process are more important as factories delivering all of the appropriate growth factors in sequence and in the amount necessary to drive the process.

An idealized osteoconductive material should be populated with marrow stem cells or another form of stem cell that can, in fact, carry out the process of providing functioning cells and providing growth factors. I do not believe that platelet-rich plasma does this purpose (it has a limited amount of some of the growth factors) and that more purified materials can do a much better job in that area.
Boden: An ideal bone graft is one that can avoid the need for harvesting autologous bone, provide the necessary mechanical resistance properties for the specific location at hand, promote rapid formation of bone and bridging/filling of the defect at least $95 \%$ of the time, and remodel over time.

I think autograft is falling out of favor as the ideal bone graft. The consequences of donor site morbidity are increasingly recorded. The healing of autograft is not consistent, especially in challenging areas such as the spine, segmental defects, or in compromised hosts (eg, smokers, steroid-dependent patients, diabetics, etc.). There is a limited supply of autograft as well.

Some healing environments in healthy hosts only need an osteoconductive scaffold to mediate bone formation. One example would be a fresh tibial metaphyseal fracture. The addition of adjuncts (PRP [platelet-rich plasma], bone marrow, concentrated bone marrow) to these osteoconductives, although popular from a marketing standpoint, has not been validated in rigorous models or clinical trials to really estimate the added benefit of the adjunct. There have been several papers to suggest that some PRPs may be inhibitory when added to autograft, at least in spine fusions.

I think the most proven adjunct to an osteoconductive scaffold would be demineralized bone matrix. These products are highly variable in terms of their osteoinductivity, but the most active brands have been well validated, in non-human primate and human studies, to have activity. 
Wang: The ideal bone graft is a graft that results in optimal fusion rates, preferably $100 \%$ without causing any donor-site morbidity. It would be easy to harvest, safe and effective, and unlimited in supply, and would not have any adverse events or side effects from single-level to multilevel fusion.

I believe that autogenous bone graft is the gold standard to which we need to compare any graft substitute. However, I would not say that it is the standard of practice currently with all the available substitutes and the increased awareness of surgeons and patients to the donor site issues. I certainly would not argue with anyone who states that it is still the gold standard or standard of care, but I think that it is now acceptable to use some type of substitute in order to avoid the donor site morbidity of autogenous iliac crest bone graft. The osteoconductive-only materials are really just scaffolding, and I think that allograft or some of the ceramics combined with bone marrow aspirates that have either concentrated or high amounts of osteoprogenitor cells are the most attractive.

I also believe that demineralized bone matrix in combination with bone marrow aspirate could be quite promising.

Question \#2. When choosing a bone graft substitute, how does the surgical indication affect your choice? Do any patient demographics affect your decision? For example, would you use the same material for a pediatric scoliosis case as you would use for an adult scoliosis case? Smoker versus non-smoker?

Boyce: I think that surgeons should consider carefully the condition of the patient, their ability to mount a healing response, and the character of the grafting site to determine if an osteoconductive material is adequate on its own. For these so-called osteoconductive materials, there is a range of performance. Some of these are remodeled completely in the host tissue, some are resorbed before they can perform their scaffolding function, and others remain at the surgical site for many months beyond the healing period.
Only by closely scrutinizing preclinical and clinical published studies can surgeons really recognize these distinctions between the materials. There is a spectrum of osteogenic potential based upon, among other things, the age, general health, and smoking status of the patient. I do believe the most successful practitioners of bone grafting will take these factors into account and will tend to increase the cell and growth factor/signal components when the patient's clinical history indicates additional risks of nonunion.
Lane: The key question here is, Is a bone graft substitute utilitarian in that it can work in any area? The answer is, No. There are essentially 6 areas that need bone graft. They can be intraosseous bone defects, anterior spine fusion, posterior spine fusion, metaphyseal fractures, diaphyseal fractures, and finally large segmental defects. Each of those requires a different material for the purpose of the regeneration and incorporation into the bone. Some of them are going to a bed rich with cells and factors and literally just need to provide a framework.
In other applications one is creating a bone across a space (fusion), where there has not been bone, or repairing a large segment of bone, including periosteum. In these circumstances, the graft has to provide all those stimuli for ultimately making a bone, and this requires a large number of cells and a high dose of growth factors to carry out the process. In addition if the vasculature has been compromised, it probably has to also contain components that will stimulate a new angiogenesis.
Boden: As I suggested earlier, the choice of bone graft substitute must be based on anatomic location and patient healing characteristics. I believe osteoinductive substitutes should be used for adult spine fusion and for healing in compromised hosts (as defined above). Pediatric scoliosis is rather unique in that purely osteoconductive materials have been proven in this application, which is less challenging because of the young age of the patients and the greater surface area of decorticated host bone and smaller gaps to bridge in a posterior spine fusion model. The rigidity of the thoracic spine is also helpful.
Wang: The surgical indication absolutely affects the choice of the bone graft substitute. Certainly there are some areas of the body that heal more readily than others. For example, the cervical spine probably has higher healing rates when compared to the lumbar spine. It also depends on how many levels we are doing; certainly single-level surgeries have a higher chance of healing than multilevel surgeries.

There are definitely some negative patient-associated factors such as medical problems, significant comorbidities such as rheumatoid arthritis or patients who are taking some medications that adversely affect the healing of bone. Smokers and patients using nicotine or patients who are not as healthy overall probably are going to be tougher to fuse and heal compared to the average person. Younger patients such as adolescent scoliotic patients are actually quite healthy and heal much more readily than an adult with comorbidities and someone who smokes. 
Question \#3. Dynamic stabilization has become a fairly new option in posterior spinal surgery. Would you use bone grafting with these systems? If you would, how do you think the flexibility in the system would affect healing?

Boyce: It seems to me that the underlying philosophies of bone grafting and of dynamic stabilization are potentially in conflict. From a purely theoretical perspective, it would seem that if the system would limit travel to within the natural strain range of bone in vivo, as described by Harold Frost, approximately 1500 to about 3000 microstrain, then there may be a positive effect on the graft, but only if the graft contains cells and has some coherency at the time the dynamic loading is applied. However, I think that the main risk in using dynamic stabilization with bone grafting is the potential to create motion great enough to disrupt the developing blood supply as the graft is being incorporated.
Lane: Dynamic stabilization is an interesting process by which you try to partially stabilize an area against torque, yet provide axial loading and bone approximation. There is no role for bone graft at that dynamic site. On the other hand, if the dynamic device is being used as a way to lead to a slow incorporation and bone formation, which is not the usual situation, then grafting could play a role. It's meant to maintain the bone in a state of controlled motion rather than no motion.
Boden: The principles of dynamic stabilization are to lessen motion in the spine or stiffen it, but not necessarily fuse the spine. Therefore it would seem that bone grafting materials would imply fusion and violate the basic premise of dynamic stabilization. That said, the dynamic concept is yet to be rigorously validated in patients and remains a hypothesis to be tested, in my opinion. As for more flexible fixation used for internal fixation during spine fusion, again, the hypothesis is that more loading of the spine fusion will result in faster healing. Again, this is a theory that has not been proven, in my estimation. I think that loading will affect remodeling of the spine fusion mass, but I am not convinced that it will increase the chances of solid connecting bone forming in the first place or prevent fibrous tissue from invading an early fusion mass and causing a nonunion.
Wang: Dynamic stabilization is a fairly new option. I do think we need to explore this area to see whether it will be effective. I think that some surgeons are using it in conjunction for fusion thinking that loading the graft may promote some faster healing. In general, I personally do not feel that these would be appropriate for fusion because I think that a rigid system would probably be better at promoting fusion; however, I am certainly not opposed to studying this aspect and evaluating the data fairly. I do not see myself using dynamic stabilization in conjunction with bone grafting for fusion at the current time, and I think dynamic stabilization is, at least in theory, designed to replace the fusion. I do think that if the literature proves that we can decrease adjacent segment disease with the use of a motion-preservation device, then there would be an advantage to using it over fusion in the right situation. However, at the current time, this is a theoretical benefit and not a proven benefit.

Question \#4. Structural allografts for interbody spine fusion are widely used. However in the past few years, polyetheretherketone (PEEK) implants are being used with greater prevalence. What are the pros/cons to structural allograft versus PEEK?

Boyce: I would think that neither structural allograft nor PEEK has all of the characteristics of an ideal interbody graft. In my mind, the search continues for the best combination of properties for this application. At their best, structural allografts have the potential to provide a template for full incorporation of the graft and to transform to the patient's bone.

Even without full incorporation, structural allografts can perform very well by remodeling at the interfaces with the endplate. Surgeons who use cortical allograft tend to favor a structural grafting member that participates in the biology of healing.
Structural allografts are extremely strong. A femoral ring used in an ALIF [anterior lumbar interbody fusion] procedure has roughly 4 times the compressive strength of the motion segment, yet on rare occasions it can still break on insertion, due to impact loading and the poor toughness of lyophilized, or freeze-dried, bone. A limitation of cortical allograft lies in the extensive period of time required for full incorporation and the potential that unremodeled islands persist. In the case of long, multilevel shaft segment grafts, the instrumentation must loadshare for a long period of time, or there is a real risk of fatigue fracture in the graft. 
Although it will never incorporate into the body, PEEK has the real advantage of offering consistent dimensions and properties as well as excellent strength characteristics. The clinicians that I've known who prefer PEEK implants tend to be reassured by the relative permanence of its properties. If revision is required, PEEK devices would likely be somewhat more difficult to revise than an allograft implant.
Perhaps even more important than the structural implant choice, whether PEEK or allograft, is that the implant allow sufficient contact area for the grafting materials to interact biologically, in direct contact with the host bone cells of the prepared endplates. A thin layer of fibrous tissue from the disc is all that is required to block a fusion.
Lane: The difficulties with allografts are that each allograft comes from an individual donor with unique properties. Different parts of the skeleton are used to prepare the allograft, and each graft is dependent upon existing bone structure. PEEK, on the other hand, is made de novo, and one PEEK material will be exactly equal to the next PEEK material in the mechanical properties, in sizing and in shape. Therefore, PEEK implants have a great advantage over allograft in reproducibility. Second, the PEEK will not be absorbed compared to the allograft, and in certain situations if you have too much growth factor, you may actually dissolve away the allograft before it has been incorporated. In that state the graft will lose some of the structural properties and may lead to malalignment of that spine. Therefore, I think that PEEK has superiority and, in fact, is being used more commonly at this time.
The allografts could be altered, however, so one could take allograft and combine it with a biochemical material, a biopolymer of some sort, which changes its strength and actually makes a new product. And this can be performed with small fragments of allograft bone, or even demineralized bone matrix mixed with a biopolymer. You could make material which could be degradable and yet have the standard properties of PEEK and give PEEK a run for the money because it would also encourage bone ingrowth. Lastly, you could put in bioglasses into a mix with an allograft, and bioglasses are known to lead to islands of bone formation and stimulate bone formation. A composite which is partially natural such as an allograft or a derivative of allograft could be mixed with a biopolymer and have superior properties and biological activity compared to PEEK. None exists at this time, but certainly the methodology is available; it just needs work.
Boden: The advantage of structural allograft is that it will eventually remodel to live bone. The disadvantage is that there is a limited supply, and due to the size and shape of bones, there are limitations to the sizes and shapes into which allograft can be made. There is also some variability in mechanical properties based on the donor although I don't see this as a major drawback. With modern processing and tissue standards I think the risk of disease transmission is not a real issue.
PEEK became popular at a time when allograft supply was limited. It has a much lower material cost, provides the companies with a higher margin, and thus has been pushed on the marketplace fairly intensely. The purported advantages are: no risk of disease transmission, unlimited supply, ability to make any size or shape with consistent mechanical properties. The primary disadvantage is that it will not remodel or allow bone to bond directly to it the way allograft will.
Wang: PEEK implants are being used, and I think that they are effective. I do not believe that they have any healing potential in and of themselves, and I think that the negative for using some type of PEEK device is that biologically they are inactive. They will certainly be there for the lifetime of the patient. They do not promote any healing whatsoever in and of themselves, but do add structural stability and have favorable radiographic imaging characteristics.
I think that the primary benefit of PEEK devices is that they are radiographically favorable. Oftentimes we can see the bone forming within the PEEK device or around the PEEK device, which can be a sign of bone healing. I think allograft certainly has the possibility of being biologically active and can be incorporated and replaced with native bone. However, the synthetic devices also have the ability to perhaps be part of a telescoping-type device, which may give a better fit, may provide more stability, and may be easier to use.

Question \#5. Interspinous devices offer a minimally invasive option for spinal stenosis. What are your thoughts on a structural allograft designed for this indication?

Boyce: Interspinous devices are probably not the best application of the properties of allograft bone, as long as the primary function is to act as a bearing surface that also maintains separation between the bony elements. For this purpose, an inert, unchanging biomaterial seems a better selection. If fusion of the spinous process were the goal, then many of the principles of posterolateral fusion could be applied here as well, and allograft could be used effectively. 
Lane: Could an allograft function very much like the interspinous processes that are currently available? The answer is, Uncertainly and probably unreliably. The current processes have a thick material property that is stronger than a bone and will not shatter, and all you have to face is the reaction of the bone to this very strong metal object. If you put in an allograft, then you have the unpredictability of not only the spinous process, but the allograft. Again, the allograft will have different properties depending on the donor, the patient, the qualities, how it is cut, how it is directed.
And it's not as reproducible. But in answer to the prior question, if one could make a hybrid, which contains some allograft and some biopolymer, you could construct a material that is reproducible and has the kind of properties that you're interested in. However, these devices function not by leading to fusion of the spinous process, but they function by stops, and we do not want to have this incorporated into the spinous process. So the virtue of the allograft is really not clear, and I would not be enthusiastic for such a device.
Boden: I have not given this much thought in the past, but I don't really understand what advantages structural allograft would offer over other materials that do not remodel or have the risk of fatigue fracture.
Wang: Interspinous devices are an area that has garnered a lot of interest lately. I do think that they are a reasonable option in properly selected patients. I think a structural allograft designed for this indication probably would do quite well in the sense that it may be able to limit motion and hold the distraction of that segment. There is always the potential that it may fracture over time with loading; that it may not be as strong as some type of metallic device; and that there may be a fusion through this area.
I do think that there are some surgeons who are using the allograft interspinous devices as a spacer, and then are billing the surgery as a fusion in order to obtain greater reimbursement for the placement of an interspinous spacer. I do not think that this is necessarily appropriate because in the truest sense of the term, they are really not performing a "fusion" in that situation. However, I think we have to look at the literature and see how the results bear out with allograft devices in this area.

Question \#6. As the SAS Journal focuses on nonfusion spinal technologies, where do you see the future of bone graft substitutes and structural allografts outside of traditional fusion procedures?

Boyce: I think that we are still in a relatively early stage for many of these technologies. If you think about how implants developed for hip and knee applications, as an analogy, these took decades and many small design and material changes to develop into the reliable products and procedures that they have become today. Currently, in the spine, fusion is still a preferred treatment for many patients, and bone grafting is still very much a part of the practice of the surgeons that I meet. But I expect motion-preserving technologies to continue to develop, outcomes to improve, and costs to decline for nonfusion treatments. As this happens, there will still be a significant place for bone grafting, though some of its roles and applications may change. Orthobiologic solutions are even now being developed that can assist in integrating an implant, repairing the annulus or entirely replacing a disc with biological tissue. Those of us who develop grafting products for surgical use need to recognize these and similar advancements and adapt our products so they can continue to be useful in changing procedures.
Lane: There are some new opportunities out there for bone graft materials, and one of them rests with the next generation for treatment of vertebral fractures. Currently the methodology is the Kyphon technique where you put in a balloon and a cement, which is not incorporated. Ultimately a little ring of resorption occurs around the cement. There is some loss of height if you follow the patients long enough over time. The vertebroplasty just injects cement, and it goes wherever it's going to go. Both of these stay in place. There is an attempt by Orthovita to develop a bioglass material called Cortoss, which has some better flowable activities and better integration within the bone, but again this is a nondegradable material.

What would be desirable is a biodegradable material that has a short-term benefit of providing great strength and in the long term leading to increased amount of bone within the vertebral body. This would be protected by drug therapy, which is the usual course of events for people with osteoporosis. I think there is now a major attempt at developing injectable allograft components mixed with biodegradable polymers, providing the appropriate mechanical property to this material. It could be quite strong, maybe melted at a relatively high temperature, but not high enough to degrade the bone. It would go into the vertebral defect and solidify. It would ultimately be incorporated and replaced by bone. This would be ideal for younger individuals where you are concerned about the material that's there for the lifetime of the patient.

You could probably develop a whole family of these devices that could also be employed in other locations at risk. It could 
also be used for stress fractures, it could be used around prostheses, in an effort to change the quality of the bone and the environment adjacent to it. I think minimally invasive techniques where you could deliver this into sites would be a great advantage particularly if it has the properties of giving good/excellent mechanical strength initially and yet be totally integrated. The difficulty with the ceramics such as Norian, for example, which works very well in Colles fracture but takes a long time to degrade and probably not in the lifetime of the

Boden: Osteoinductive bone growth factors could play a role in more immediate fixation of motion preservation implants. Another role will be in filling the large bone defects that will patient. Another difficulty is that it's very brittle so you cannot drill instrumentation into it. What you'd like is some material that has the ability to solidify, be drillable, and then ultimately be replaced in 2-4 years, so that it is totally integrated within the patient. I think this is where a great future would be open and allow for interbody manipulation such as percutaneous osteotomies of the fractured bodies, and then jack them up and put this in. You could use it for scoliosis and other kinds of deformities and also for fractures at this particular time.
Wang: I think that bone graft substitutes will become more and more the standard. I believe we will look towards better fusion rates without any donor site morbidities and towards something that is more cost effective. So I think that, at least in the short term, bone graft substitutes are going to continue to be displayed prominently in the area of biologics.

I also think that we are developing nonfusion spinal technologies, but some of these devices will fail. There are many patients that probably are not candidates for nonfusion technologies and will need fusions. Thus, I do believe that undoubtedly be left when the motion preservation devices are removed, since they will eventually fatigue or fail. fusion will always be needed as a potential option in spinal surgery, and I do think that we will continue to need to strive for better fusion surgeries with improved bone grafting options. I do think that perhaps bone graft substitutes are becoming more scientific with more data proving their efficacy. In addition, the research in this area is advancing rapidly, and we are learning more and more about biologics in general which may lead to advances and applications outside of fusion. Perhaps modified forms of these bone graft substitutes may be used as scaffolds for tissue engineering, perhaps for cartilage or for disk repair. I do think that the future will be very exciting in this area. 